\title{
FATORES ASSOCIADOS À INTERNAÇÃO PROLONGADA NAS ADMISSÕES PELA URGÊNCIA E EMERGÊNCIA
}

Daniel Silva MORAES ${ }^{1}$

Noemea Mendes CORDEIRO

Adélia Dayane Guimarães FONSECA ${ }^{3}$

Luís Paulo SOUZA e SOUZA ${ }^{4}$

Carla Silvana de OLIVEIRA e SILVA ${ }^{5}$

Joanilva Ribeiro LOPES ${ }^{6}$

\begin{abstract}
${ }^{1}$ Enfermeiro, Mestrando em Ciências da Saúde pela Universidade Estadual de Montes Claros. Minas Gerais, Brasil. silvamoraes.daniel@gmail.com

${ }^{2}$ Enfermeira, Graduada pelas Faculdades Santo Agostinho de Montes Claros. Minas Gerais, Brasil. adeliadayane@yahoo.com.br

${ }^{3}$ Enfermeira, Doutoranda em Ciências da Saúde pela Universidade Estadual de Montes Claros, Professora do Departamento de Enfermagem das Faculdades Santo Agostinho de Montes Claros. Minas Gerais. adeliadayane@yahoo.com.br

${ }^{4}$ Enfermeiro, Doutorando em Saúde Pública pela Universidade Federal de Minas Gerais, Professor do Departamento de Medicina da Universidade Federal de São João del Rei, Campus Dom Bosco. Minas Gerais, Brasil. luis.pauloss@hotmail.com

${ }^{5}$ Enfermeira, Doutora em Ciências pela Universidade Federal de São Paulo, Professora do Departamento de Enfermagem da Universidade Estadual de Montes Claros. Minas Gerais, Brasil. profcarlasosilva@gmail.com

${ }^{6}$ Enfermeira, Mestre em Ciências pela Universidade Federal de São Paulo, Professora do Departamento de Enfermagem da Universidade Estadual de Montes Claros. Minas Gerais, Brasil. joanilval@ fasa.edu.br
\end{abstract}

Recebido em: 10/12/2016 - Aprovado em: 10/12/2017 - Disponibilizado em: 30/12/2017

RESUMO: Objetivou-se estimar os fatores envolvidos na permanência hospitalar prolongada de pacientes admitidos em caráter de urgência e emergência. Coorte prospectiva, com 125 pacientes admitidos na urgência e emergência de um hospital em Minas Gerais. A coleta dos dados foi feita nos prontuários eletrônicos, em 2014. Os pacientes foram selecionados e acompanhados por 60 dias até a ocorrência dos desfechos "alta" ou "óbito". Os pacientes foram divididos em curta e longa permanência hospitalar. Como longa permanência, consideraram-se aqueles pacientes que ultrapassaram 4 dias de internação. Odds Ratio (OR) e seus respectivos Intervalos de Confiança de 95\% (IC95\%) foram ajustados pela regressão logística. Testaram-se diferenças entre média pelo teste qui-quadrado. A média de dias de permanência foi 8,1 ( $\mathrm{DP}=8,9)$, sendo que a incidência de longa permanência foi de 56,8\%. Mantiveram-se independentemente associadas à longa permanência: presença de comorbidades (OR=3,4; IC95\% 1,3-9,0); diagnósticos no decorrer da internação (OR=8,8; IC95\% 2,0 - 40,0); uso de dispositivos invasivos (OR=3,1; IC95\% 1,2-8,5); sepse (OR=7,0; IC95\% 1,5-31,1); permanecer mais dias internados no pronto socorro; permanecer mais tempo com dispositivos invasivo; fazer uso de antibióticos por mais dias; submeter a mais procedimentos; estar nas faixas etárias de 0 a 20 ano e acima de 41 anos; apresentar infecções relacionadas à assistência à saúde. Estas informações evidenciam que algumas variáveis são passíveis de serem monitoras e modificáveis. Assim, rastrear os fatores associados à longa permanência poderá servir para indicar inconformidades aos gestores e profissionais, de modo que sejam delimitados planos de melhorias da assistência prestada.

Palavras-chave: Internação Hospitalar. Tempo de internação. Qualidade da Assistência à Saúde.

\section{FACTORS ASSOCIATED WITH PROLONGED INPATIENT ADMISSIONS BY THE URGENCY AND EMERGENCY}

\footnotetext{
ABSTRACT: Aimed to estimate the factors involved in prolonged hospital stay of patients admitted on the basis of urgency and emergency. Prospective cohort, with 125 patients admitted on emergency and emergence of a hospital in the State of Minas Gerais. The data collection was made in electronics, in 2014. Patients were selected and 680 
accompanied by 60 days until the occurrence of the outcomes "high" or "death". The patients were divided into short and long hospital stay. How long stay, those patients were considered beyond 4 days of hospitalization. Odds Ratio (OR) and their respective 95\% confidence intervals (95\% CI) were adjusted by logistic regression. Differences between average were tested by the Chi-square test. The average days of stay was $8.1(\mathrm{DP}=8.9)$, the incidence of long permanence was $56.8 \%$. Remained independently linked to long stay: presence of Comorbidities $(\mathrm{OR}=3.4$; 95\% CI 1.3 9.0); diagnoses in the course of hospitalization $(\mathrm{OR}=8.8 ; 95 \% \mathrm{CI} 2.0-40.0)$; use of invasive devices $(\mathrm{OR}=3.1 ; 95 \% \mathrm{CI}$ 1.2-8.5); sepsis (OR=7.0; 95\% CI 1.5-31.1); stay more days admitted in the emergency room; stay longer with invasive devices; make use of antibiotics for several days; undergo more procedures; be in the age group of 0 to 20 year and over 41 years; present healthcare-related infections. This information show that some variables are likely to be monitors and modifiable. So, trace the factors associated with the long stay could serve to indicate non-conformities to managers and professionals, so that they are marked improvements of the assistance plans.

Keywords: Length of Stay. ; Hospitalized patients; Quality of health care.

\section{INTRODUÇÃO}

A internação é um recurso usado na tentativa de recuperar a saúde do indivíduo. Quando este período se prolonga demasiadamente, há incremento de custos e redução da rotatividade de leitos, desta forma, a permanência hospitalar prolongada serve como indicador da eficiência e qualidade do serviço hospitalar (RUFINO et al., 2012).

Mesmo sendo uma alternativa indispensável para a recuperação da saúde, a internação pode representar riscos ao paciente. Os eventos adversos são danos relacionados à assistência à saúde, sendo que cada uma em 10 admissões hospitalares, um evento adverso pode ser observado e, na metade dos casos, as causas são evitáveis. Aproximadamente, um terço dos eventos adversos causam danos reais. Os erros mais comuns são os relacionados à prescrição e administração de medicamentos; procedimentos cirúrgicos; diagnósticos; transferência de pacientes; troca de informações; falhas na operação de máquinas e equipamentos; infecções hospitalares (WACHTER, 2010).
Dias, Martins e Navarro

(2012) avaliaram 6.179.859 internações no Sistema de Informação Hospitalar do Sistema Único de Saúde (SIH-SUS), tendo observado que o tempo de internação foi maior na clínica médica quando comparada à cirúrgica. Além disso, constaram que os pacientes que sofreram os eventos adversos "úlcera de pressão", "sepse" e "pneumonia" apresentaram os maiores tempos médios de permanência. Aqueles que desenvolveram úlcera de pressão permaneceram internados cerca de oito dias a mais que a média dos que desenvolveram outros eventos adversos. Complementarmente, o tempo de permanência, o gasto hospitalar e a taxa de mortalidade foram maiores nas internações com registro de resultado adverso, quando comparado aquelas sem ocorrência de erros.

É importante destacar que o tempo de internação pode ainda ser prolongado por questões administrativas como falta de recursos de custeio e equipamentos quebrados. Ocasionalmente, alguns pacientes são internados alguns dias antes ou permanecem além do necessário para garantir 
a hospitalização ou realizarem exames complementares e especializados (ROQUE; MELO, 2011).

Autores esclarecem que quanto mais tempo os pacientes que permaneceram internados, maiores são as chances de sofrerem eventos adversos, podendo ser citados aqueles relacionados a medicamentos e ocorrência de infecções nosocomiais (ROQUE; MELO, 2011; RUFINO et al., 2012). Quando se avalia os fatores relativos à condição de cada paciente, Rufino et al. (2012) encontraram em seu estudo que permaneceram mais tempo internados os homens, as pessoas com diabetes mellitus, com renda menor que cinco salários mínimos e os idosos.

Conhecer sobre os fatores envolvidos no tempo de permanência dos pacientes tornase fundamental, no sentindo de permitir a identificação de erros na gestão hospitalar e falhas da assistência, além de servir de subsídio para melhorias de qualidade do serviço de saúde prestado (ROQUE; MELO, 2011; RUFINO et al., 2012).

Diante do exposto, esta pesquisa objetivou estimar os fatores envolvidos na permanência hospitalar prolongada de pacientes admitidos em caráter de urgência e emergência em um hospital de Minas Gerais, Brasil.

\section{METODOLOGIA}

Trata-se de uma coorte prospectiva, realizada em um Hospital filantrópico na cidade de Montes Claros, norte de Minas Gerais. O Hospital conta com 325 leitos e é referência para alta e média complexidade em todo o norte de Minas Gerais. O seu serviço de urgência e emergência conta com um Pronto Socorro (PS) e Pronto Atendimento (PA) com funcionamento ininterrupto de 24 horas, contando com as especialidades de cardiologia, neurologia, clínica médica, ginecologia e obstetrícia, pediatria, cirurgia geral e ortopedia.

A seleção da população foi feita durante uma semana de 01 de julho de 2014 até 07 de julho de 2014, estando elegíveis para participação no estudo todos pacientes atendidos em urgência e emergência pelas portas de entrada do PS e PA. Os critérios de inclusão foram: ser internado após o atendimento em qualquer setor do hospital, incluindo PS e PA. Como critérios de exclusão, consideraram-se: pacientes que permaneceram no hospital por menos de 24 horas; pacientes atendidos para assistência gineco obstétrica. Assim, participaram do estudo 125 pacientes.

A coleta de dados foi realizada no hospital, utilizando o prontuário informatizado, permitindo gerar relatórios no sistema. O formulário de coleta de dados foi criado pelos próprios pesquisadores e, uma semana antes da coleta, realizou-se um pré- 
teste em 20 prontuários. Após seleção dos participantes, fez-se o acompanhamento de, no máximo, 60 dias de todos os pacientes até a ocorrência dos desfechos "alta", "óbito" ou "transferência externa".

Os pacientes foram divididos em dois grupos: em curta permanência hospitalar e em longa permanência. Para esta classificação, estabeleceu-se o ponto de corte de 4 dias de internação, de modo que pacientes que ficaram internados até 4 dias contados a partir da admissão foram classificados como curta, aqueles que ultrapassaram os 4 dias entraram na longa permanência. Este ponto de corte foi estabelecido através da mediana e da distribuição do número de dias de permanência de toda a amostra.

Os dados foram organizados e analisados no programa Statistical Package for the Social Sciences (SPSS) versão 20.0. A variável dependente foi tempo de permanência hospitalar (curta x longa). Como variáveis independentes, consideraram-se: sexo; idade; município de residência; encaminhado por Serviço de Atendimento Móvel de Urgência (SAMU) ou Bombeiros; porta de entrada; tipo de internação; especialidade médica; custeio da internação; classificação no Protocolo de Manchester; permanência no PS (sim/não e número de dias); diagnóstico de internação; comorbidades; novos diagnósticos ao longo da internação; cirurgias e procedimentos hemodinâmicos realizados (sim/não e número de procedimentos); uso de dispositivos invasivos (sim/não e quais); uso de antibióticos (sim/não e número de dias); desenvolvimento de infecção relacionada à assistência à saúde (IRA's); desenvolvimento de sepse; internação em Unidade de Tratamento Intensivo (UTI); úlcera por decúbito e desfecho do paciente (óbito ou alta/transferência).

Para as variáveis "diagnóstico", "comorbidades" e "diagnósticos no decorrer da internação", as informações relatadas nos prontuários foram agrupadas em categorias com base nos capítulos do Código Internacional de Doenças (CID-10). Na variável "infecção relacionada à assistência à saúde", não foram seguidos critérios específicos do Serviço de Controle de Infecção Hospitalar (SCIH), entretanto, seguiu-se a partir da leitura criteriosa dos prontuários em busca de relatos que evidenciassem infecções decorrentes da hospitalização, bem como início dos sinais de infecção após 48 horas da admissão; uso de antibióticos; foco infeccioso relacionado a algum procedimento; culturas positivas para floras de microrganismos característicos do hospital; e a própria descrição em prontuário da natureza da infecção. Para a variável "sepse", seguiram-se os mesmos critérios de protocolo já implantado na instituição. Quanto ao uso de dispositivos invasivos, foram considerados para este estudo os dispositivos: sonda vesical de demora (SVD); 
sonda nasoenteral ou oroentérica (SNE); tubo orotraqueal (TOT); cateter venoso central de curta permanência (CVC). Para os pacientes que usaram mais de um dispositivo, foram criadas categorias e foi considerado o maior número de dias entre os dispositivos.

Realizaram-se análises descritivas, por meio de medidas absolutas e relativas, as frequências foram expressas por porcentagem e as quantidades por média, desvio padrão (DP), mediana, mínimo e máximo. A análise bivariada foi conduzida para avaliar a associação bruta das variáveis independentes de interesse com a longa permanência, por meio da regressão logística simples. Aquelas variáveis que apresentaram significância estatística inferior a $20 \%$ durante a análise bivariada foram consideradas na elaboração do modelo final de regressão logística múltipla. As estatísticas provenientes dos modelos construídos nas análises multivariadas foram: odds ratio (OR), intervalo de confiança (IC) de 95\%, estimados pela regressão logística múltipla. Além disso, foi realizado um teste de comparação entre as médias dos dados quantitativos, por meio do teste do Qui-quadrado, a partir do fator permanência (longa ou curta) do paciente. Nesse sentido, as variáveis consideradas foram: idade do paciente (em anos), previsão de permanência, número de dias internado no PS/PA, número de dias com dispositivo invasivo, número de dias que tomou antibiótico e o número de procedimentos realizados. O nível de significância adotado nas análises foi de $5 \%$.

Este trabalho foi aprovado pelo Comitê de Ética em Pesquisa das Faculdades Integradas Pitágoras de Montes Claros, com parecer número 27418214.2.0000.5109.

\section{RESULTADOS}

Dos 125 pacientes acompanhados, 54 $(43,2 \%)$ apresentaram curta permanência e 71 $(56,8 \%)$ longa permanência. A média de dias de internação foi 8,1 (DP = 8,9), mediana de 5, mínimo de 1 dia e máximo de 47.

A Tabela 1 mostra o perfil dos pacientes descrito pelas variáveis categóricas e estratificado pelos grupos de curta e longa permanência.

Tabela 1 - Perfil dos pacientes segundo grupos de curta e longa permanência. Montes Claros, Minas Gerais, 2014

\begin{tabular}{|c|c|c|c|c|c|c|}
\hline \multirow[t]{2}{*}{ Variável } & \multicolumn{2}{|c|}{ Curta permanência } & \multicolumn{2}{|c|}{ Longa permanência } & \multicolumn{2}{|c|}{ Total } \\
\hline & $\mathbf{N}$ & $\%$ & $\mathbf{N}$ & $\%$ & $\mathbf{N}$ & $\%$ \\
\hline \multicolumn{7}{|l|}{ Sexo } \\
\hline Masculino & 31 & 24,8 & 44 & 35,2 & 75 & 60 \\
\hline Feminino & 23 & 18,4 & 27 & 21,6 & 50 & 40 \\
\hline \multicolumn{7}{|l|}{ Faixa etária } \\
\hline 0 a 10 anos & 9 & 7,2 & 15 & 12 & 24 & 19,2 \\
\hline 11 a 20 anos & 3 & 2,4 & 8 & 6,4 & 11 & 8,8 \\
\hline 21 a 30 anos & 11 & 8,8 & 4 & 3,2 & 15 & 12 \\
\hline
\end{tabular}


31 a 40 anos

41 a 50 anos

51 a 60 anos

Mais de 60 anos

Forma de atendimento

Sistema Único de Saúde - SUS

Particular

Porta de entrada

Pronto Socorro

Pronto Atendimento

Tipo de internação

Clínica

Cirúrgica

Permanência no Pronto Socorro maior que 24 horas

Sim

Não

Dispositivos invasivos

Com dispositivos

Sem dispositivos

Diagnósticos no decorrer da internação

Sim

Não

Comorbidades

Sim

Não

Sepse

Sim

Não

Infecção Relacionada à

Assistência à Saúde

Sim

Não

Antibioticoterapia

Sim

Não

Procedimentos

Sim

Não

Internação em Unidade de

Tratamento Intensivo

Sim

Não

Lesão de pele

Sim

Não

Desfecho

Alta

Óbito
10

3

5

14

37

17

35

19

23

31

11

43

6

48

2

52

6

48

2

52

0

54

35

19

32

22
8

2,4

4

11,2

29,6

13,6

28

15,2

18,4

24,8

8,8

34,4

4,8

38,4

1,6

42,6

4,8

38,4

1,6

41,6

0

43, 2

28

15,2

25,6

17,6

$1 \quad 0,8$

53

1

53

51

3
42,4

6

65

0,8

42,4

4

67

40,8

2,4

\section{2,4}

4

7,2

21,6

44

12,8

36,8

36,8
20

29,6

27,2

60

65

13

10,4

6,4

$14 \quad 11,2$

$40 \quad 32$

$92 \quad 73,6$

$33 \quad 26,4$

$81 \quad 64,8$

$44 \quad 35,2$

48

$\begin{array}{llll}27,2 & 65 & 52\end{array}$

22

17,6

33

$39,2 \quad 92 \quad 73,6$

26,4

$\begin{array}{lll}16 & 26 & 20,8\end{array}$

$\begin{array}{lll}40,8 & 99 & 79,2\end{array}$

$\begin{array}{lll}14,4 & 20 \quad 16\end{array}$

$42,4 \quad 105 \quad 84$

$\begin{array}{lll}16,8 & 27 & 21,6\end{array}$

$\begin{array}{lll}40 & 98 & 78,4\end{array}$

$12 \quad 17 \quad 13,6$

$\begin{array}{lll}44,8 & 108 & 86,4\end{array}$

56

\section{9}

62

7,2

49,6

$9 \quad 7,2$

11692,8

$32 \quad 75 \quad 60$

$\begin{array}{lll}24,8 & 50 & 40\end{array}$

$32 \quad 72 \quad 57,6$

$\begin{array}{lll}24,8 & 53 & 42,4\end{array}$

31

4,8

52

$7 \quad 5,6$

11894,4

3,2

53,6

$5 \quad 4$

$120 \quad 96$

A média de idade foi 42,6 anos (DP =

clínica cirúrgica 16,8\%; neurologia 15,2\%;

$28,4)$ e a mediana foi 40 anos. Maioria residia cardiologia $12 \%$; ortopedia $11,2 \%$; nefrologia na cidade de Montes Claros (63,2\%). A 1,6\%; ginecologia, urologia e oncologia $0,8 \%$ cada. A informação de encaminhamento pelo SAMU ou Bombeiros foi coletada para 89 dos 
admitidos, destes, 21 foram levados ao hospital por um destes serviços. Quanto à triagem do Protocolo de Manchester, 34,4\% ficaram sem informação; para os demais, o maior número de classificações foi laranja $(29,6 \%)$ e amarelo (24,8\%). Os principais fluxos de classificação foram: mal estar em adultos $(n=13)$; trauma maior $(n=8)$; hemorragia digestiva e dor abdominal em adultos $(\mathrm{n}=4)$. Após a admissão, 26,4\% dos pacientes ficaram internados por, no mínimo, um dia no PS, sendo que a média de dias foi de 3,6 (DP = 2,9), mediana de 3 e máximo de 16 dias. Nenhum paciente ficou internado no PA.

Após a internação, o desfecho de 114 dos pacientes foi alta hospitalar ou a transferência externa. Em relação ao desfecho óbito, 11 o apresentaram, sendo que as causas foram assim distribuídas: 2 por choque séptico; 3 por complicações decorrentes de acidente vascular encefálico; 2 por doença cardíaca; 1 por politraumatismo, insuficiência respiratória, falência renal e hepática, respectivamente. Para 21,6\% dos pacientes foi atribuída também alguma comorbidade, sendo a principal a hipertensão arterial $(n=16)$, seguida do diabetes $(n=7)$, doença renal $(n=4)$, imobilidade crônica $(n=4)$.

Quanto às variáveis coletadas no decorrer da internação, $16 \%$ receberam um novo diagnóstico enquanto estavam internados, sendo os mais comuns as infecções, doenças do aparelho respiratório e doenças do aparelho digestivo. Em relação ao uso de dispositivos invasivos, 20,8\% usaram algum durante a internação, sendo que a maioria destes $(n=10)$ usou apenas sonda vesical de demora. Em relação à infecção relacionada à assistência à saúde, 9 pacientes foram identificados com esta infecção. $60 \%$ fizeram uso de antibióticos durante a internação, sendo que a média de dias de uso de antibiótico foi 6,21 (DP = 8). Em 17 pesquisados foram encontrados critérios para sepse, sendo que 10 tiveram apenas sepse e 7 evoluíram para sepse grave e choque séptico.

A análise bivariada foi conduzida para avaliar a associação bruta das variáveis independentes de interesse com a longa permanência, por meio da regressão logística simples. Aquelas variáveis que apresentaram significância estatística inferior a $20 \%$ durante a análise bivariada foram consideradas na elaboração do modelo final de regressão logística múltipla. Assim, será apresentado aqui somente o modelo final.

A Tabela 2 apresenta os resultados da análise multivariada por regressão logística múltipla para associação entre variáveis de interesse e longa permanência. 
Tabela 2 - Modelo final pós-análise de Regressão Logística múltipla. Montes Claros, Minas Gerais - Brasil, 2014.

\begin{tabular}{|c|c|c|c|c|c|c|}
\hline \multicolumn{3}{|c|}{ Variável } & \multicolumn{2}{|c|}{ OR (IC 95\%) } & \multicolumn{2}{|c|}{ p valor } \\
\hline \multirow{2}{*}{\multicolumn{3}{|c|}{$\begin{array}{l}\text { Dispositivos invasivos } \\
\text { Sem dispositivos }\end{array}$}} & \multirow{3}{*}{\multicolumn{2}{|c|}{$\begin{array}{c}1,00 \\
3,10(1,2-8,5)\end{array}$}} & \multirow{3}{*}{\multicolumn{2}{|c|}{0,024}} \\
\hline & & & & & & \\
\hline \multirow{2}{*}{\multicolumn{3}{|c|}{$\begin{array}{l}\text { Com dispositivos } \\
\text { Diagnósticos no decorrer da internação }\end{array}$}} & & & & \\
\hline & & & \multirow{3}{*}{\multicolumn{2}{|c|}{$\begin{array}{c}1,00 \\
8,80(2,0-40,0)\end{array}$}} & \multirow{3}{*}{\multicolumn{2}{|c|}{0,005}} \\
\hline \multirow{2}{*}{\multicolumn{3}{|c|}{$\begin{array}{l}\text { Não } \\
\text { Sim }\end{array}$}} & & & & \\
\hline & & & & & & \\
\hline \multicolumn{3}{|l|}{ Comorbidades } & \multirow{3}{*}{\multicolumn{2}{|c|}{$\begin{array}{c}1,00 \\
3,40(1,3-9,0)\end{array}$}} & \multirow{3}{*}{\multicolumn{2}{|c|}{0,016}} \\
\hline & & & & \\
\hline Sim & & & & & & \\
\hline \multicolumn{3}{|l|}{ Sepse } & & \multirow{2}{*}{\multicolumn{2}{|c|}{0,012}} \\
\hline \multicolumn{3}{|l|}{ Não } & & & & \\
\hline Sim & & & & & & \\
\hline \multicolumn{3}{|l|}{ Antibioticoterapia } & \multicolumn{2}{|c|}{$7,00(1,5-31,1)$} & & \\
\hline Não & & & 1,0 & & 0,33 & \\
\hline Sim & & & $0,70(0$, & & & \\
\hline Lesão de pele & & & & & & \\
\hline Não & & & 1,0 & & 0,30 & \\
\hline Sim & & & $3,20(0,3$ & ,1) & & \\
\hline Internação em Unidade de & Catamento Inter & & & & & \\
\hline Não & & & 1,0 & & 0,14 & \\
\hline Sim & & & $4,90(0,6$ & 1,9) & & \\
\hline Procedimento cirúrgico e h & nodinâmico & & & & & \\
\hline Não & & & 1,0 & & 0,74 & \\
\hline Sim & & & $0,90(0$, & & & \\
\hline Mantiveram-se & independente & & invasiv & $(\mathrm{OR}=3$ & ; IC 95\% 1,2-8 & ) e sepse \\
\hline associadas à longa pern & Inência preseı & & $(\mathrm{OR}=$ & ; IC 95\% & $, 5-31,1)$. & \\
\hline $\begin{array}{l}\text { comorbidades }(\mathrm{OR}=3, \\
\text { diagnósticos no decorrer }\end{array}$ & $\begin{array}{l}\text {; IC } 95 \% 1,3 \\
\text { la internação }\end{array}$ & & resulta & $\begin{array}{l}\text { Tabela } 3 \\
\text { do teste }\end{array}$ & $\begin{array}{l}\text { por sua vez, ap } \\
\text { e comparação c }\end{array}$ & $\begin{array}{l}\text { senta os } \\
\text { médias. }\end{array}$ \\
\hline 8,$8 ;$ IC $95 \% 2,0-40,0$ & uso de dispo & & & & & \\
\hline $\begin{array}{l}\text { Tabela } 3 \text { - Teste de com } \\
\text { Montes Claros, Minas G }\end{array}$ & $\begin{array}{l}\text { aração de mé } \\
\text { ais - Brasil, 2 }\end{array}$ & & o fator & rmanênci & hospitalar long & curta. . \\
\hline Variável & Permanência & $\mathbf{N}$ & Média & $\begin{array}{c}\text { Desvio } \\
\text { padrão }\end{array}$ & $\begin{array}{l}\text { Diferença entre } \\
\text { as médias }\end{array}$ & p valor \\
\hline Idade & Longa & 71 & 44,7 & 29,9 & 4,8 & 0,353 \\
\hline & Curta & 54 & 39,9 & 26,2 & & \\
\hline Dias de internacão no & Longa & 22 & 4,2 & 3,4 & 1,6 & 0,045 \\
\hline Pronto Socorro & Curta & 11 & 2,6 & 0,9 & & \\
\hline Dias com dispositivo & Longa & 20 & 7,8 & 5,6 & 5,1 & 0,041 \\
\hline invasivo & Curta & 06 & 2,7 & 1,0 & & \\
\hline Dias em uso de antibiótico & Longa & 40 & 10,1 & 9,4 & 8,3 & $<0,001$ \\
\hline & Curta & 35 & 1,8 & 0,9 & & \\
\hline Número de procedimentos & Longa & 40 & 2,1 & 2,6 & 1,0 & 0,018 \\
\hline invasivos & Curta & 32 & 1,1 & 0,2 & & \\
\hline
\end{tabular}


Encontrou-se

associação

estatisticamente significativa entre longa permanência e pacientes que permaneceram mais dias internados do pronto socorro; que permaneceram mais tempo com dispositivos invasivo; que usaram antibióticos por mais dias; que se submeteram a procedimentos.

Dados não apresentados nas Tabelas evidenciaram que houve associação entre faixas etárias de 0 a 20 anos e acima de 41 anos com a longa permanência $(p=0,002)$. $O$ mesmo ocorreu para os pacientes que desenvolveram IRA's $(p=0,007)$.

\section{DISCUSSÃO}

Por apresentar diversas variáveis, serão discutidas aquelas julgadas pelos autores como as mais relevantes.

Talvez devido à natureza dispersa da população, esta pesquisa apresentou uma média de tempo de internação menor do que outras. Em estudo que buscou investigar os fatores que interferem no tempo de internação em uma enfermaria de clínica médica na Paraíba, a amostra foi composta por 48 pacientes e a média de tempo de internação foi de 20,9 dias (RUFINO et al., 2012). Já no estudo de Silva et al. (2013), a média de permanência hospitalar foi de 11 dias $( \pm 10,89)$.

Quanto à forma de atendimento, a maioria dos pacientes acompanhados foi atendida pelo Sistema Único de Saúde (SUS), entretanto, não foi observada associação com a longa permanência. Em pesquisa realizada para verificar possíveis diferenças entre o tempo de permanência hospitalar dos pacientes submetidos à cirurgia de revascularização do miocárdio entre aqueles cujo atendimento foi pelo SUS e entre os custeados por outros convênios privados ou particulares, identificou-se que para os atendidos pelos convênios/particular, o número de dias de internação hospitalar, internação em terapia intensiva e tempo de pós-operatório foram maiores do que para os clientes do SUS. Dentre os 2840 clientes, $91,1 \%$ pertenciam ao grupo SUS e $7,9, \%$ ao grupo de convênios/particular (SILVA et al., 2013). A divergência entre os achados deste estudo com o de Silva e colaboradores pode ser explicada pela natureza diversa desta população, visto que, provavelmente, um número significativo de pacientes atendidos pelos convênios/particular admitidos pela urgência tinham problemas mais fáceis de serem resolvidos que os usuários do Sistema Único de Saúde.

Uma situação recorrente nos hospitais com serviço de urgência e emergência por demanda espontânea é a superlotação das unidades de pronto socorro, forçando à improvisação de leitos extras por meio de macas pelos corredores, o que torna o ambiente inadequado para os pacientes que ficam submetidos às condições insatisfatórias (acomodados em locais de grande fluxo de pessoas, altos índices de ruído e luminosidade 
constante) (OHARA; MELO; LAUS, 2010). Nesta pesquisa, 26,4\% dos pacientes ficaram internados no pronto socorro por pelo menos um dia, sendo que a média foi de 3,6 dias (DP = 2,9), e um paciente apresentou 16 dias de permanência neste setor. A maioria se enquadrou no grupo de longa permanência. Não houve associação estatística quando aplicado o teste de Qui-quadrado entre longa permanência e permanência o PS, entretanto, no teste de médias, foi significativa a comparação de médias de dias de internação no setor entre os grupos de curta e longa permanência $(p=0,045)$.

Foi evidenciada associação da longa permanência com a presença de comorbidades, sendo corroborada por estudo desenvolvido na Arábia Saudita (ALEITHAN; AMIN; ROBERT, 2011). Segundo os mesmos autores, pacientes vítimas de ataque vascular encefálico (AVE), com diabetes mellitus e/ou hipertensão arterial sistêmica tiveram significativamente maior permanência hospitalar quando comparados com outros que também sofreram AVE, mas que não possuíam tais doenças crônicas. Hipertensão arterial e diabetes também foram as principais comorbidades encontradas neste estudo, dos 27 pacientes que apresentaram alguma doença secundária 21 ficaram internados por mais de 4 dias. Resultado diferente foi encontrado no estudo na Paraíba, Brasil, em que hipertensão arterial e diabetes mellitus não foram significativas na definição do tempo de internação (RUFINO et al., 2012).,

Em relação à faixa etária (categorizada), evidenciou-se associação entre faixas etárias de 0 a 20 anos e acima de 41 anos com a longa permanência, dada este corroborado por outras pesquisas. No estudo de Rufino et al. (2012), foram evidenciadas associações entre as variáveis idade, mudança da dor e escolaridade. Segundo este mesmo estudo, a maioria dos pacientes que permaneceram mais de dez dias internados eram mais velhos, tinham menos anos de estudo e não apresentaram melhora ou pioraram o seu quadro de dor. Ao avaliar o perfil de internações de idosos em um hospital público, Motta, Hansel e Silva (2010) descobriram que o tempo de permanência hospitalar de $85 \%$ da clientela atendida esteve em torno de 30 dias. Para os autores, o idoso permanece mais tempo hospitalizado que as pessoas de outras faixas etárias, o que gera necessidade de cuidados especiais para este grupo. Sobre a relação com a faixa abaixo de 20 anos, não foram encontrados estudos que discutem estes achados.

A ocorrência de IRA's foi tida como significativa frente à longa permanência, o mesmo ocorreu para a sepse. O uso de antibiótico não apresentou significância no teste de regressão logística, porém, no teste de comparação de médias mostrou-se significativo $(\mathrm{p}<0,001)$. Uma hipótese para isso pode ser devido ao grande número de 
pacientes que fizeram uso de antibiótico para profilaxia e pertencem ao grupo de curta permanência.

A prevenção, tratamento e controle das IRA's são um desafio para as autoridades governamentais, institucionais e profissionais de saúde por constituir grave problema de saúde e ser causa considerável de mortalidade hospitalar (BATISTA, 2012). Na pesquisa de Lima, Andrade e Haas (2007), as taxas de infecção hospitalares na Unidade de Terapia Intensiva do Hospital das Clínicas da Faculdade de Medicina de Ribeirão Preto foram maiores em pacientes com maior tempo de intenção. Os valores encontrados aqui pesquisa apontam que a média de dias de internação hospitalar entre os pacientes que contraíram IRA's foi de 28 dias; 20,5 dias na UTI. Já nos pacientes sem IRA's, a média foi de 11,2 dias na internação geral e 4,5 dias de internação na UTI. Dos pacientes com IRA's, a permanência foi maior naqueles colonizados por bactérias multi-droga-resistentes, com média de 39 dias; pacientes infectados por bactérias sensíveis permaneceram, aproximadamente, 8 dias a menos.

Esperava-se que outras variáveis se mantivessem associadas ao desfecho, tais como internação em UTI e lesão de pele. Entretanto, a frequência do desfecho nas categorias de algumas variáveis explicativas foi baixa, dificultando as comparações. Além disso, a maneira como as variáveis foram avaliadas e categorizadas pode ter influenciado os resultados.

\section{CONCLUSÃO}

Como fatores que se mantiveram independentemente associadas à longa permanência, tiveram-se presença de comorbidades; diagnósticos no decorrer da internação; uso de dispositivos invasivos; sepse; permanecer mais dias internados no pronto socorro; permanecer mais tempo com dispositivos invasivo; fazer uso de antibióticos por mais dias; submeter a mais procedimentos; estar nas faixas etárias de 0 a 20 ano e acima de 41 anos; apresentar IRA's.

Estas informações evidenciam que algumas variáveis são passíveis de serem monitoras e modificáveis. Assim, rastrear os fatores associados à longa permanência poderá servir para indicar inconformidades aos gestores e profissionais assistenciais, de modo que sejam delimitados planos de melhorias e organização, otimizando recursos e alcançando a excelência na prestação de cuidados à saúde.

Destaca-se que este estudo apresenta algumas limitações, citando o uso de dados secundários; ausência de outras informações importantes e consideras em outras pesquisas com mesmo objetivo; restrição a uma única instituição. Assim, estudos futuros que superem estas limitações devem ser realizados. 


\section{REFERÊNCIAS}

AL-EITHAN, M.H.; AMIN, M.; ROBERT,

A.A. The effect of hemiplegia/hemiparesis, diabetes mellitus, and hypertension on hospital length of stay after stroke.

Neurosciences, v.16, n. 3, p.253-256, 2011.

BATISTA, O.M.A. Representações sociais de enfermeiras sobre a infecção hospitalar: implicações para o cuidar prevencionista.

Revista de Enfermagem UERJ, v.20, n.4, p.500-506, 2012.

DIAS, M.A.E.; MARTINS, M.; NAVARRO, N. Rastreamento de resultados adversos nas internações do Sistema Único de Saúde.

Revista de Saúde Pública, v.46, n.04, p.719729, 2012.

LIMA, M.E.; ANDRADE, D.; HAAS, V. J. Avaliação prospectiva da ocorrência de infecção em pacientes críticos de unidade de terapia intensiva. Revista Brasileira de Terapia Intensiva, v.19, n.3, p.342-347, 2007.

MOTTA, C.C.R.; HANSEL, C.G.; SILVA, J. Perfil de internações de pessoas idosas em um hospital público. Revista Eletrônica de Enfermagem, v.12, n.3, p.471-7, 2010. Disponível em:

<https://www.fen.ufg.br/fen_revista/v12/n3/p df/v12n3a08.pdf>. Acesso em: 09 dez. 2016.

OHARA, R.; MELO, M.R.A.C.; LAUS, A.M. Caracterização do perfil assistencial dos pacientes adultos de um pronto socorro.

Revista Brasileira de Enfermagem, v.63, n.5, p.749-754, 2010.

OLIVEIRA, A.C.; KOVNER, C.T.; SILVA, R.S. Infecção hospitalar em unidade de tratamento intensivo de um hospital universitário brasileiro. Revista LatinoAmericana de Enfermagem, v.18, n.2, P.97104, 2010.

ROQUE, K.E.; MELO, E.C.P. Tempo de internação e a ocorrência de eventos adversos a medicamentos: uma questão da enfermagem. Escola Anna Nery, v.15, n.3, p.595-601, 2011.

RUFINO, G.P. et al. Avaliação de fatores determinantes do tempo de internação em clínica médica. Revista Brasileira de Clínica Médica, v.10, n.4, p.291-297, 2012.

SILVA, G.S. et al. Avaliação do tempo de permanência hospitalar em cirurgia de revascularização miocárdica segundo a fonte pagadora. Revista da Associação Médica Brasileira, v.59, n.3, p.248-253, 2013.

WACHTER, R.M. Compreendendo a segurança do paciente. Porto Alegre: Artmed, 2010. 